\title{
Eugenik, soziale Wohlfahrt, Frieden
}

Jakob Tanner

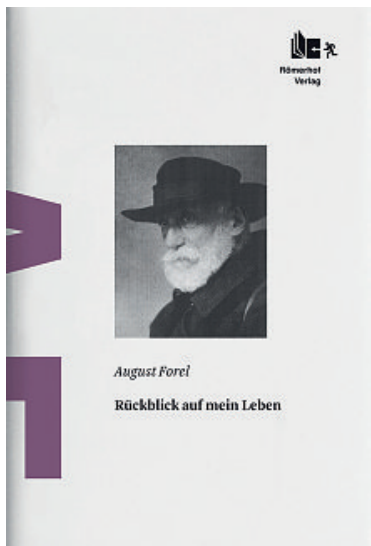

August Forel Rückblick auf mein Leben. Mit einem Essay von Richard Müller.

Zürich: Römerhof Verlag; 2010. 406 Seiten. $47.90 \mathrm{CHF}$. ISBN 978-3-905-89405-9

Korrespondenz: Prof. Dr. phil. Jakob Tanner Forschungsstelle für Sozialund Wirtschaftsgeschichte Universität Zürich Rämistr. 64 CH-8001 Zürich

jtanner@hist.uzh.ch
1935, vier Jahre nach seinem Tod, erschien August Forels «Rückblick auf mein Leben» gleichzeitig im Zürcher Europa Verlag und in der Büchergilde Gutenberg. Die bebilderte Publikation enthält eine facettenreiche Beschreibung seines Lebens, die mit dem Hinweis auf sein «persönliches Testament» endet. Im Anhang wurde diese 1912 nach einem Schlaganfall verfasste und 1921 erweiterte «selbstverfasste Grabrede» abgedruckt; der Text wurde anlässlich der Kremation von Forels Leiche im Juli 1931 in Lausanne von seinem Sohn verlesen. 1947 erschien die Autobiographie in zweiter Auflage, was auf eine ungebrochene Nachfrage nach diesen pointiert verfassten, immer wieder selbstironisch gebrochenen Lebenserinnerungen hindeutet. Forel schreibt selbstkritisch.

Dennoch bleibt er bis ans Lebensende der festen Überzeugung, dass die Menschheit auf der breiten Heerstrasse des wissenschaftlichen Fortschritts in die lichte Zukunft einer «rationellen menschlichen Zuchtwahl» gehen würde. Forel schildert in farbiger Sprache die katastrophalen Zustände, die er als junger Psychiater in Schweizer Anstalten vorfand, er stellt sich als preisgekrönten Ameisenforscher, als Neuronentheoretiker und Hypnotiker, als «aktiven Sozialisten» und Kämpfer für die Gleichberechtigung der Geschlechter dar. Dabei zieht er eine gerade Linie von der «Eugenik der Besten, der Sterilisierung der Schlechteren» zur «sozialen Wohlfahrt aufgrund eines supranationalen Friedens».

Nun ist dieses Werk erneut aufgelegt worden wobei der Römerhof Verlag ankündigt, er würde «die Autobiographie August Forels veröffentlichen». Eine Neuedition müsste anders vorgestellt werden. Nur im kleingedruckten Impressum wird darauf hingewiesen, die «Originalausgabe von 1935» sei «sprachlich leicht überarbeitet und modernisiert» worden. Weitere Angaben zur philologischen Textkritik und Editionstechnik fehlen, und es findet sich auch kein Hinweis darauf, wieso auf die eindrücklichen Illustrationen darunter das Forel-Porträt von Oskar Kokoschka aus dem Jahre 1910 - verzichtet wurde. Das Werk vermag editorischen Standards, die an eine Neuausgabe gestellt werden müssen, in verschiedener Hinsicht nicht zu genügen. Die Chance wurde verpasst: Eine wissenschaftlich fundierte Herausgabe des «Rückblicks auf mein Leben» steht nach wie vor aus.

Der Begleitessay hätte dieses Problem mit Hinweisen auf die Entstehungs- und Rezeptionsgeschichte des Werks entschärfen können. Richard Müller, ehemaliger Direktor der Schweizerischen Fachstelle für Alkohol- und andere Drogenprobleme, beschränkt sich allerdings darauf, den Inhalt der Autobiographie nach Themen sortiert zusammenzufassen. Das Welt- bild und Lebenswerk, das für Forel durchaus aus einem Guss war, wird in eine gute und eine schlechte Seite zweigeteilt. Daraus resultiert ein «Zwiespalt»: Forel war «ingeniöser Wissenschaftler, Sozialreformer und Philanthrop einerseits und zugleich rabiater Verfechter der Eugenik andererseits». Dass der Essay unter dem Titel «Der Wahrheit verpflichtet» segelt, mit einer starken Geste der «Bewunderung» beginnt und in die Feststellung einmündet, Forel bleibe «ein starker und glaubwürdiger Anwalt seiner Wissenschaft», drückt die Präferenz des Autors im «Bewertungskonflikt» aus. Die bipolare normative Hintergrundfolie führt dazu, dass Vorwürfe immerzu gleich wieder relativiert werden. Obwohl die Frage, ob Forel «ein Rassist» sei, «zwar bejaht» wird, darf man «Forel nicht einfach als Rassisten abtun und in die Nähe des Nationalsozialismus rücken». Forels Formulierungen mögen «zwar schauerlich tönen, doch zu seiner Zeit hätten wohl die meisten Ärzte diese Worte unterschrieben». Der Sozialdarwinismus, der Forels Bestseller «Die sexuelle Frage» von 1905 durchdringt, «darf die Tatsache nicht vernebeln, wie revolutionär seine Sexuallehre ist». Obwohl «Forels Theorie der Keimverderbnis (...) nicht aufrechterhalten» werden kann, «nimmt die Theorie die heute als Embryopathie bezeichnete Schädigung der Kinder durch den Alkoholkonsum der Eltern vorweg». Richard Müller hat begriffen, dass man heute über Forel nicht mehr hagiographisch schreiben kann - und so versucht er, zu retten, was zu retten ist.

\section{Das Weltbild und Lebenswerk, das für Forel aus einem Guss war, wird in eine gute und eine schlechte Seite zweigeteilt}

Die Wissenschafts- und Psychiatriegeschichte hat indessen seit einiger Zeit andere Interpretationen vorgelegt, die eine solche unproduktive Problemstellung hinter sich lassen. In einem von Anton Leist herausgegebenen Sammelband (Auguste Forel - Eugenik und Erinnerungskultur, 2006) wird gezeigt, wie sich die Bewertung Forels im 20. Jahrhundert mit dem soziokulturellen Wertewandel verschoben hat. Verschiedene Studien von Stefan Kühl und Matthias M. Weber verweisen auf die Bedeutung wissenschaftlicher und politisch-persönlicher Netzwerke bei der Propagierung der Eugenik und der Rassenideologie. 
Auch wenn sich Forel deutlich links positionierte, haben Schüler von ihm die nationalsozialistische Weltanschauung vertreten, was einige komplexe Fragen nach ideellen und wissenschaftlichen Wirkungszusammenhängen aufwirft. In den Studien von Paul Weindling wiederum wird betont, dass sich die Eugenik nie als akademische Fachdisziplin durchsetzen konnte, sondern als eine von aufstrebenden Mittelschichten getragene soziale Bewegung verstanden werden muss. Diese Charakterisierung ist zentral für die Untersuchung der phänomenalen Medienwirkung damaliger Erfolgsautoren wie August Forel. Geschlechtergeschichtliche Untersuchungen (etwa von Regina Wecker) haben die Frage gestellt, wieso in der Schweiz der psychiatrische Zugriff auf den Frauenkörper besonders dezidiert ausfiel, und dabei gezeigt, dass der Forelsche Einsatz für Sterilisierung trotz seiner Forde- rung nach einer Gleichstellung des weiblichen Geschlechts in einer stark männerdominierten und patriarchalen Gesellschaft signifikant auf Kosten der Frauen ausgehen musste. Wiederum andere Studien (Stefan Kühl und Maria A. Wolf) weisen nach, dass sich die Rationalisierung der Fortpflanzung, die Forel mit seiner Sexualwissenschaft anstrebte, in ein weiteres Bild einer verwissenschaftlichen Gesellschaft einfügt. Die Eugenik ist ein Anwendungsgebiet eines die politischen Lager übergreifenden Kontrollgedankens, welcher die wirtschaftliche Produktivität gleichermassen wie die Volksgesundheit zu erhöhen anstrebte. Richard Müller versucht allerdings nicht einmal eine ansatzweise Auseinandersetzung mit dem Forschungsstand. Die wissenschaftliche Neugierde fehlt. Somit bleibt auch der Essay hinter den Erwartungen des interessierten Lesers zurück. 